\title{
BRECHAS DE GÉNERO EN EL MERCADO LABORAL: UNA MIRADA CRÍTICA ANTE EL HORIZONTE 2030
}

\author{
Sarai Rodríguez González \\ Universidad de La Laguna \\ sarodri@ull.edu.es
}

\section{RESUMEN}

La participación de las mujeres en el mercado de trabajo ha experimentado aumentos significativos en las últimas décadas, persistiendo, pese a ello, importantes desigualdades por causa de género que han venido a cristalizar en las distintas facetas en las que se materializa la brecha de género: acceso y permanencia al mercado de trabajo; promoción profesional; salarios y pensiones; y usos del tiempo y roles familiares. En el marco de la Agenda sobre Desarrollo Sostenible 2030 de Naciones Unidas, este trabajo aborda una revisión de las principales causas y factores determinantes de la persistente desigualdad en el mercado de trabajo, realizando un análisis crítico de la efectividad de algunas de las medidas puestas en marcha hasta ahora para combatirla y proponiendo, en su caso, fórmulas alternativas o complementarias para seguir avanzando en su erradicación.

PAlabras Clave: brecha de género, trabajo, igualdad, discriminación, independencia económica.

GENDER GAPS IN THE LABOR MARKET:

A CRITICAL LOOK A THE 2030 HORIZON

\section{Abstract}

Women's participation in the labour market has experienced significant increases in recent decades. Nevertheless, important gender inequalities persist that come to crystallize in the different facets in which the gender gap materializes: access and permanence in the labour market; professional promotion; salaries and pensions; and uses of time and family roles. Within the framework of 2030 Agenda for Sustainable Development adopted at the United Nations, the his paper provides a review of the main causes and determinants of persistent inequality in the labour market, making a critical analysis of the effectiveness of some of the measures implemented so far to combat it, and proposing, where appropriate, alternative or complementary formulas to its eradication.

KEYWORDS: gender gap, work, equality, discrimination, economic independence. 


\section{LA PARTICIPACIÓN DE LA MUJER EN EL MERCADO DE TRABAJO}

La participación femenina en el mundo del trabajo ha registrado aumentos significativos en las últimas décadas no obstante, con carácter general, la participación femenina en la fuerza laboral se ha mantenido por debajo de la masculina y los mercados siguen estando divididos por géneros (Grau y Rodríguez, 2015: 13). De hecho, la Comisión ha puesto de manifiesto que en la mayoría de los países, la participación femenina en el mercado laboral continúa manteniéndose por debajo de su potencial a pesar de los progresos y avances que las mujeres han experimentado en su cualificación profesional en los últimos años (Eurofound, 2016), sin que se haya conseguido cumplir el objetivo planteado por la Estrategia Europa 2020 para el empleo consistente en garantizar una tasa de empleo total del $75 \%$ para hombres y mujeres en el conjunto de la Unión Europea.

Desde el año 2014, tal como se refleja en los datos de Eurostat correspondientes al segundo trimestre de 2017, la tasa de ocupación en Espańa se ha ido recuperando a un ritmo más intenso que el de la media de la Unión Europea, recobrando casi el 90\% del empleo indefinido perdido durante la crisis. Específicamente atendiendo a la diferencia de la recuperación por sexos, ésta está siendo algo más intensa entre los hombres (60\% del empleo creado). Concretamente, la tasa de empleo de los hombres superó a la femenina en 11,96 puntos porcentuales en el tercer trimestre de 2017. Con todo, las mujeres, que perdieron menos empleo durante la crisis, se sitúan ahora en máximos, superando los 8,5 millones de afiliadas a la Seguridad Social. De forma conexa, por lo que se refiere a la brecha de género en el desempleo, aunque el número de mujeres desempleadas registradas en los Servicios Públicos de Empleo ha descendido casi un 20\% desde septiembre del año 2013, en octubre de 2017 representan el 57,85\% del total de personas que solicitan empleo.

Sin embargo, y a pesar de que en la actualidad trabajan 596000 mujeres más que en 2005 (un 7,8\% más), la incorporación femenina al mercado laboral apenas ha representado un aumento de la tasa de ocupación de la mujer de 2,8 puntos en estos once años, teniendo en cuenta que la población femenina entre los 16 y los 65 años aumentó en este periodo en 334911 mujeres.

Con todo, la Organización Internacional del Trabajo ha insistido en que no es suficiente con alcanzar mayor presencia femenina en el mercado de trabajo, sino que son primordiales políticas que se dirijan a mejorar la calidad de los empleos y a aprovechar el talento y capacidad de las mujeres. Este reto, plasmado desde 2013 como una de las siete Iniciativas del Centenario de la OIT contenidas la Memoria del Director General titulada Ante el centenario de la OIT: Realidades, renovación y compromiso tripartito, persigue, en definitiva, hacer realidad la igualdad de oportunidades y de trato en el mercado de trabajo y en la sociedad, permitiendo alcanzar el objetivo del trabajo decente para las mujeres (OIT, 2013). A mayor abundamiento, «lograr la igualdad entre los géneros y empoderar a todas las mujeres y las niñas» constituye una prioridad independiente en la Agenda 2030 de Desarrollo Sostenible (ODS número 5) adoptada en la cumbre especial de Naciones Unidas el 25 y 26 de 
septiembre de 2015 en Nueva York ${ }^{1}$ (Ramos, 2017a: 246-249). En consonancia con este objetivo, la Estrategia Española para el Empleo 2017-2020² considera que uno de los retos principales del mercado de trabajo español es «mejorar la activación y la inserción del colectivo de mujeres, así como sus posibilidades de acceso, mantenimiento y promoción profesional en el empleo, de forma que las condiciones de trabajo sean efectivamente iguales».

Profundizando en la realidad en el seno del mercado laboral, se detecta que la presencia de las mujeres en el trabajo a tiempo parcial, la economía informal y en puestos de trabajo mal remunerados sigue siendo una constante (Ramos, 2017a: 245). Sectores y actividades como agricultura, trabajo a domicilio, trabajo asistencial y trabajo doméstico representan espacios de actividad económica en los que las mujeres encuentran un lugar para proveerse de ingresos. Concretamente, por lo que se refiere a la feminización del trabajo a tiempo parcial (OCDE, 2013: 216 y ss.), cabe destacar que en el en el periodo 2010-2015 -según los datos de la Encuesta de Población Activa del INE-, la participación de las mujeres en el trabajo a tiempo parcial se ha elevado de un $22,7 \%$ en 2010 a un $25,2 \%$ en 2015. Las mujeres representan el $72 \%$ del total de las personas con jornada a tiempo parcial. En 2016, un $26,6 \%$ de las mujeres de entre 25 y 54 ańos empleadas y con un hijo trabajaban a tiempo parcial. Entre los hombres en idéntica situación, eran solamente el 5,7\%. Ellas, por tanto, multiplican por cuatro el porcentaje de su representación en el trabajo a tiempo parcial.

La indagación en las causas que motivan la opción por el trabajo a tiempo parcial confirma el carácter «involuntario» de este tipo jornadas laborales para las mujeres (en 2015 el 80,1\% de las mujeres no pudo encontrar un trabajo a tiempo completo, el $12,7 \%$ de las mujeres tenían que cuidar niños o adultos y el 5,9\% manifestaban tener obligaciones familiares o personales [Grau y Rodríguez, 2015: 65]. A la luz de estas cifras, hay quienes advierten que «la utilización del trabajo a tiempo parcial como una forma de permiso parental, mientras persista la tendencia a ser una opción casi exclusivamente femenina, tiene como inevitable consecuencia la segregación del mercado de trabajo y es perniciosa para la calidad y estabilidad del empleo de la mujer, suponiendo una traba a su pleno desarrollo profesional» (Ramos y Blázquez, 2007: 143).

En otro orden de consideraciones, el acceso de las mujeres a puestos de máxima responsabilidad y cargos de dirección en la gestión empresarial permanece registrando niveles muy bajos de participación ${ }^{3}$. En consecuencia, el «techo de cris-

1 «Transformar nuestro mundo: la Agenda 2030 para el Desarrollo Sostenible», de 18 de septiembre de 2015. Emitido por la Comisión de Derechos de la Mujer e Igualdad de Género (2014/2217(INI). El objetivo 5 de los ODS viene a sustituir el tercero de los ocho objetivos de Desarrollo del Milenio (ODM) de Naciones Unidas correspondientes al periodo 2000-2015.

2 Decreto 1032/2017, de 15 de diciembre, por el que se aprueba la Estrategia Española de Activación para el Empleo 2017-2020 (BOE del 16).

3 Aunque la situación ha mejorado en los últimos años, las cifras más recientes muestran -según el Informe de Eurostat con motivo del Día Internacional de la Mujer 2017- que solo el 35 
tal» supone uno de los principales aspectos que dificultan la integración equitativa de las mujeres en el mercado de trabajo (OCDE, 2013: 250 y 251). Al respecto, en 2013, el Informe del Fondo Monetario Internacional titulado «Las mujeres, el trabajo y la economía: beneficios macroeconómicos de la equidad de género» llamaba la atención sobre el hecho de que las mujeres representan más de la mitad de la población mundial y, pese a ello, su contribución a la actividad económica medida, al crecimiento y bienestar se encuentra muy por debajo de su potencial, con las importantes consecuencias macroeconómicas que ello conlleva (FMI, 2013). Por su parte, la OIT abordaba el problema del techo de cristal en un Informe titulado «La mujer en la gestión empresarial. Cobrando impulso» (2015), en el que se revelaron datos de máximo interés y se pusieron de manifiesto interrogantes acerca de por qué las mujeres no alcanzan a ocupar puestos de máxima responsabilidad en la gestión empresarial a pesar del aumento de su formación y cualificación profesional (Ramos, 2015: 99-103). Poner luz sobre esos interrogantes es una cuestión que exige atención inaplazable, pues permitirá diseñar y formular propuestas que combatan esta realidad y permitan aprovechar económicamente el talento de las mujeres en concordancia con el Objetivo 5,5 de los ODS, referido a la necesidad de «velar por la participación plena y efectiva de las mujeres y la igualdad de oportunidades de liderazgo a todos los niveles de la adopción en la vida política, económica y pública».

En el ámbito europeo, la Comisión ha confirmado en su Compromiso estratégico para la igualdad de género 2016-20194 el objetivo de que haya al menos una representación del $40 \%$ de mujeres en los cargos de dirección no ejecutivos de las empresas con cotización en Bolsa. Igualmente, el Consejo ha reconocido la importancia de la igualdad en la participación de mujeres y de hombres en todas las escalas y ámbitos de la toma de decisiones, y ha incluido esta cuestión entre las áreas prioritarias del Pacto por la Igualdad de Género 2011-20205.

\section{LA BRECHA DE GÉNERO EN SALARIOS Y PENSIONES}

Como se ha venido diciendo, el acceso generalizado de las mujeres al sistema educativo y la mejora de su cualificación se ha considerado uno de los principales logros en materia de igualdad de oportunidades (OCDE, 2013: 202 y ss.; CES-España, 2011: 75 y ss.). Pese a ello, aún existe segregación ocupacional horizontal y vertical en función del sexo y persisten igualmente injustificables disparidades de carácter salarial (OCDE: 2013: 224 y ss.). Aunque las cifras correspondientes a la

por ciento de los puestos directivos en empresas de la Unión Europea son ocupados por mujeres. Del total de 7,3 millones de cargos de dirección que hay en la UE, 2,6 son mujeres. Además, las mujeres que ocupan puestos directivos en la UE obtienen un salario un $23,4 \%$ inferior a la media que los hombres, lo que significa que las jefas de empresa ganan un promedio de 77 céntimos por cada euro que gana un hombre por hora.

${ }^{4}$ SWD (2015) 278 final.

5 2011/C 155/02. 
brecha salarial entre hombres y mujeres varían en los distintos países de la UE, por término medio, las mujeres de la UE ganan alrededor de un 16\% menos por hora que los hombres. En España, según el último Informe de UGT, titulado «La falta de políticas de igualdad en el empleo incrementa la brecha salarial», las mujeres cobran de media 6000 euros menos al año por hacer un trabajo de igual valor que los hombres. Una brecha que, según el informe «Brecha salarial y Techo de cristal tienen nombre de mujer", elaborado por el sindicato de Técnicos del Ministerio de Hacienda (Gestha), ha aumentado en los últimos años desde el 22,55\% de $2010 \mathrm{al}$ $23,25 \%$ de 2014, y al 29,1\% en 2016; y que se agrava si se computan los complementos salariales, respecto a los cuales las mujeres ingresan un $44 \%$ menos que los hombres (CC. OO., 2018). A ello podemos unir que las mujeres representan el 70\% de la población asalariada con ingresos inferiores al salario mínimo interprofesional, mientras que solo representan el $28 \%$ de las personas que ganan más de ocho veces este salario mínimo interprofesional (CC. OO., 2018). Estas cifras no hacen más que explicitar la desigualdad en la valoración del trabajo que realizan las mujeres (Ramos, 2017a: 245).

De este modo, la promoción de la independencia económica de hombres y mujeres mediante la reducción de las disparidades de ingresos constituye uno de los compromisos contenidos en el Pacto por la Igualdad de Género (2011-2020), así como una de las áreas prioritarias de actuación del Compromiso estratégico para la igualdad entre mujeres y hombres 2016-2019-y que estaba presente desde la Estrategia para la igualdad entre mujeres y hombres 2010-2015 de la Comisión Europea y que contó con el impulso ofrecido por la Recomendación de la Unión Europea de 7 de marzo de 2014 sobre el refuerzo del principio de igualdad de retribución entre hombres y mujeres a través de la transparencia ${ }^{7}$, considerándola como «un requisito previo para que mujeres y hombres puedan controlar sus vidas y tengan auténtica libertad para elegir». Asimismo, en el Informe "Adecuación de las pensiones en la Unión Europea 2010-2050», la Comisión y el Comité de Protección Social reconocieron que una importante dimensión del reto de la suficiencia de las pensiones está ligada al género. La importancia de este reto ha sido subrayada de forma reciente por la ONU al indicar que el fortalecimiento económico de la mujer es esencial para alcanzar los Objetivos de Desarrollo Sostenible para 2030. De forma particular, la meta 8,5 de los ODS se refiere a la garantía de la igualdad de remuneración por trabajo de igual valor, que sirve para empoderar a las mujeres, reducir la pobreza, crear condiciones de trabajo decentes y la igualdad de género?.

${ }^{6}$ COM (2010) 491 final.

2014/114/UE

8 Pension Adequacy in the European Union 2010-2050, informe preparado conjuntamente por la Dirección General de Empleo, Asuntos Sociales e Inclusión de la Comisión Europea y el Comité de Protección Social, 23 de mayo de 2012.

${ }^{9}$ Una actuación para alcanzar esta meta ha cristalizado en septiembre de 2017 con la creación por parte de OIT, ONU-Mujeres y la OCDE de la Coalición sobre la igualdad de remuneración para desafiar la igualdad salarial (EPIC). 
Las desigualdades salariales por razón de género contagian inevitablemente al sistema de pensiones, donde se manifiesta con mayor virulencia la brecha de género. El hecho de que las mujeres obtengan menos ingresos a lo largo de su vida conduce a pensiones más bajas y a un mayor riesgo de pobreza en la tercera edad (OCDE, 2013: 315). La menor participación de las mujeres en el mercado laboral por entrar más tarde en él y protagonizar el disfrute de las excedencias, reducciones de jornada y empleos a tiempo parcial provoca que el sistema público de pensiones perpetúe y amplíe la brecha salarial entre sexos. La traslación de esta afirmación a cifras reales permite constatar que los hombres mayores de 64 ańos han trabajado una media de 43,4 años, mientras que las mujeres de esa franja de edad solo lo han hecho un promedio de 12,8 años, la cifra más baja de Europa (Comisión Europea, 2014: 2; Comisión Europea, 2013).

Según los datos existentes para la UE-27, las mujeres perciben pensiones que, de media, son un 38,5\% inferiores a las de los hombres. Además, de media en la UE-27 el 63\% de las mujeres se encuentran en el rango de las personas que perciben las pensiones más bajas; situándose España entre los países que tienen una mayor incidencia de las pensiones más bajas para las mujeres y solo un $1 \%$ de las mujeres perciben pensiones elevadas (Tinios et al., 2015: 44). A ello se suma, como agravante, por un lado, que un conjunto de mujeres al haberse incorporado de manera más tardía al mercado de trabajo tienen una menor participación en el ámbito de las prestaciones contributivas y mayor en el nivel no contributivo; y, por otro, que más de un tercio de las mujeres mayores en Europa no reciben ningún tipo de pensión (CES, 2011: 202 y ss.).

La dificultad en la erradicación de la brecha de género en las pensiones obedece -en concordancia con el Informe de la Comisión Europea titulado «Men, Women and Pensions-2015"- a que se trata de una materia de gran complejidad, apreciándose notables diferencias entre de los países de la UE tanto cuantitativas -oscilando entre el $46 \%$ en unos países y el $4 \%$ en otros- como cualitativas -apreciándose en cada país distinta incidencia de los factores desencadenantes-. Con todo, la brecha de género en las pensiones y el fomento de la independencia económica de las personas se han posicionado como objetivos prioritarios en la agenda política de la UE, de ahí que el 19 de junio de 2015, en Consejo de Ministros de Empleo y Asuntos Sociales, los países de la Unión Europea acordaran un texto de Conclusiones sobre Igualdad de oportunidades de ingresos para las mujeres y hombres: cerrando la brecha de género en las pensiones ${ }^{10}$, en el que respaldaron la puesta en marcha de un plan de acción global para controlar y corregir la brecha de género basado en un planteamiento transversal de las políticas de género a lo largo de la trayectoria vital de una persona, combinando medidas preventivas y medidas paliativas (apartado 18). Por este mismo enfoque transversal e integrador es por el que se ha decantado el Parlamento Europeo en junio de 2017 en su Propuesta de Resolución sobre la necesidad de una estrategia de la Unión para eliminar y prevenir la brecha de género en materia 
de pensiones, donde resalta que la brecha en las pensiones «es un reflejo de la gran cantidad de desigualdades que experimentan las mujeres a lo largo de la vida y en su carrera profesional» y que, por lo tanto, «hace falta seguir una estrategia global y generalizada para combatirla» (Rodríguez González, 2017b: 103).

De este modo, se consolida como idea fuerza la interconexión del conjunto de circunstancias padecidas por las mujeres a lo largo de su trayectoria vital y profesional en el mercado de trabajo. Tal es así que la brecha de género en las pensiones se convierte en una problemática cuya solución no depende de mejorar los derechos de las pensiones sino también de mitigar las interrupciones de las carreras de cotización de las mujeres, reducir la brecha salarial con los hombres, mejorar la conciliación de trabajo y vida privada o, entre otros objetivos, aumentar la tasa de empleo de las mujeres y su presencia en puestos de máxima responsabilidad y liderazgo.

Tratando de solucionar las disfunciones y perjuicios que provoca en las mujeres (en sus carreras profesionales y en su acceso al sistema de protección social), el pasado jueves 22 de febrero de 2018 -coincidiendo con la conmemoración del Día Europeo de la Igualdad Salarial- el Pleno del Congreso dio luz verde, con el voto a favor de todos los grupos de la oposición y la abstención del PP, a la tramitación de la Ley de Igualdad Retributiva de Unidos Podemos ${ }^{11}$. La norma pretende erradicar la brecha salarial adoptando medidas de distinta naturaleza, entre otras: a) la publicación de los sueldos medios por parte de las empresas; b) que las nóminas de los empleados incluyan información del promedio del salario distribuido por género; y c) que el convenio contenga de forma obligatoria los criterios específicos que justifiquen una determinada retribución o complemento. Además, tratando de garantizar el efectivo cumplimiento de esta disposición, la norma propone la creación de una unidad especial que fiscalice el cumplimiento de la norma dotada de competencias para inspeccionar y sancionar a las empresas.

A la proposición de Ley presentada por Unidos Podemos en octubre de 2017, se sumaba el 20 de febrero de este año la del PSOE ${ }^{12}$. Ambas propuestas coinciden en cinco planteamientos: a) la necesidad de rendir cuentas a los trabajadores a través de la publicación anual de sueldos con desglose por sexos (en un libro de registro en el caso del PSOE; o en las nóminas según Podemos) y; b) la realización de auditorías salariales; c) el desglose de infracciones y sanciones para los empleadores que no cumplan la normativa; d) la necesidad de crear órganos que controlen la efectividad de la ley (el PSOE propone la creación de Oficina Nacional de Lucha contra la discriminación por razón de sexo en la empresa y la ocupación dentro de la Inspección de Trabajo; Podemos apuesta por la creación de tres organismos diferentes en distintos

${ }^{11}$ El texto de la proposición de Ley de Igualdad retributiva entre mujeres y hombres de Unidos Podemos puede consultarse en http://www.congreso.es/public_oficiales/L12/CONG/BOCG/B/BOCG-12-B-171-1.PDF.

${ }^{12}$ El texto íntegro de la Proposición de Ley para garantizar la igualdad de trato y de oportunidades entre mujeres y hombres en materia retributiva (122/000184) puede consultarse en https://www. ecestaticos.com/file/3aca882d62ca9396b574df0c2561a599/1519051745-proposicio-n-de-ley-igualdad-retributiva_maquetado.pdf. 
niveles: una Unidad especializada en materia de igualdad de trato en todas las Inspecciones Provinciales de Trabajo y Seguridad Social, una Dirección General para la Igualdad Retributiva dependiente de la secretaria de Estado de Servicios Sociales y una Comisión interministerial específica); e) la importancia de que el Instituto de la Mujer colabore con la Inspección de Trabajo, aunque Unidos Podemos propone añadidamente la atribución a este organismo de nuevas competencias específicas destinadas a asegurar la efectividad de la norma.

\section{LA BRECHA DE GÉNERO EN LOS USOS DEL TIEMPO Y EN LOS ROLES FAMILIARES}

La brecha de género en la participación en el mercado de trabajo, en los salarios y en las pensiones tiene una multiplicidad de elementos desencadenantes, apareciendo, si bien de manera muchas veces camuflada, como principal factor detonante el hecho de que las vidas laborales de las mujeres se hallen más afectadas por las responsabilidades familiares que las de los hombres. Según los datos del Índice de igualdad de género 2017, a nivel europeo, en lo que respecta al uso del tiempo por parte de las mujeres y los hombres, solo uno de cada tres hombres dedica tiempo diariamente a la cocina y las tareas domésticas, frente a la práctica totalidad de las mujeres (79\%) (EIGE, 2017). Se trata de una realidad que hereda las consecuencias de la distribución sexual del trabajo, toda vez que «la mujer se incorpora al mercado laboral desde un espacio de distribución de roles totalmente consolidado, la mujer cumple una función reproductiva y de soporte familiar, mientras que el hombre cumple una función productiva y se convierte en el sostén económico de la familia. Esta distribución de roles o asignación de funciones tiene unas connotaciones que sobrepasan la estructura familiar, en la medida en que forman parte de la organización social y de las estructuras económicas en todas sus dimensiones» (Tortuero, 2006: 113).

A pesar de que el modelo de familia ha cambiado considerablemente en las últimas décadas y de que se ha generalizado el modelo de doble sustentador, las mujeres continúan responsabilizándose de las labores de cuidado y de la organización del hogar familiar (OCDE, 2013: 270). La asunción casi en exclusiva de las responsabilidades familiares obliga a muchas mujeres, en el peor de los escenarios, a renunciar por completo a su trabajo para atender el cuidado de menores o familiares dependientes, lo que puede suponer que sus carreras profesionales se vean truncadas; mientras, en otras ocasiones, las mujeres deciden asumir el coste derivado de las interrupciones en sus carreras de cotización, lo que puede impedirles cotizar lo suficiente o no hacerlo en los años finales de su etapa antes de la jubilación, con la consecuente merma en el cálculo global de la pensión (Tinios et al., 2015: 47 y ss., Rodríguez González, 2017b: 100). Por lo que se refiere a la frecuencia de las interrupciones de las carreras laborales de las mujeres, como dato concluyente cabe indicar que, según datos del MESS sobre prestaciones por maternidad o paternidad y excedencias por cuidado familiar correspondientes a 2017, publicados en enero 2018, en el año 2017 se registraron 55133 excedencias por cuidado de hijos, menores acogidos u otros 
familiares, de las que 49934 correspondían a solicitudes de mujeres (90,6\%) y 5199 a solicitudes de hombres. Se trata de datos que no solo reflejan que el cuidado de hijos/as o familiares dependientes penaliza las carreras de las mujeres, sino que la economía de los cuidados recae en las mujeres.

De este modo, la desigualdad en el reparto de responsabilidades familiares dentro del núcleo familiar (Fernández-Lozano, 2017); la escasez de una oferta de servicios de cuidado asequibles, adecuados y de calidad acorde a los Objetivos de Barcelona $2002^{13}$; y la pervivencia de modelos de organización empresarial basados en la presencia en el puesto de trabajo a tiempo completo y con horarios rígidos constituyen factores que limitan extremadamente las posibilidades reales de las mujeres de acceso, permanencia y promoción en el mercado de trabajo en igualdad de oportunidades que los hombres (OCDE: 2013: 220; Eurofound, 2016). De ahí que las mujeres con responsabilidades que desean o necesitan incorporarse o permanecer en el mercado laboral consideren como una medida adecuada para conseguir el equilibrio trabajo-familia optar por el trabajo a tiempo parcial, que permite una mayor flexibilidad horaria y que, además, suele estar vinculado generalmente a trabajos no declarados o sumergidos (OCDE, 2013: 204 y ss., y 302 y ss.).

Tratando de solventar los obstáculos que la asunción de responsabilidades de cuidado supone para la presencia y permanencia de las mujeres en el mercado laboral y con un enfoque de la política de conciliación de la UE más acorde a su naturaleza de derecho fundamental (Rodríguez González, 2016), el Objetivo número 8.4 de los ODS concretamente se refiere a la garantía de «reconocer y valorar los cuidados no retribuidos y el trabajo doméstico mediante la prestación de servicios públicos, la provisión de infraestructuras y la formulación de políticas de protección social, así como mediante la promoción de la responsabilidad compartida en el hogar y la familia, según proceda en cada país». De modo parecido, la Propuesta de Resolución del Parlamento Europeo (junio 2017) sobre la necesidad de una estrategia de la Unión para eliminar y prevenir la brecha de género en materia de pensiones ${ }^{14}$ incluye, entre las actuaciones que componen la Estrategia de la Unión para eliminar y prevenir la brecha de género en materia de pensiones, la mejora del equilibrio entre la vida profesional y la personal mediante permisos de maternidad, paternidad y parentales como «instrumentos necesarios e indispensables», esquemas flexibles y "créditos por cuidados» de maternidad, paternidad o cuidado de otros familiares; así como la necesidad de que existan estructuras de prestación de servicios para niños, mayores o personas dependientes que permitan aliviar la carga.

Junto a estas líneas de actuación, no cabe desdeñar la necesidad de visibilizar y dar valor al trabajo no remunerado de los cuidados que se realiza en los hogares. La configuración en términos jurídicos del trabajo doméstico en el hogar y su valoración

13 El Consejo Europeo de Barcelona, de 15-16 de marzo de 2002, se ocupa de la necesidad de remover las dificultades para la contratación de la mano de obra femenina y la necesidad de fomentar los servicios de cuidado de niños [COM (2008)638].

14 2016/2061(INI). 
económica permitiría superar la situación de tradicional dependencia de la mujer respecto al varón, al tiempo que se superaría la división tradicional de roles asociados al género (Rodríguez-Piñero, 2004: 117 y 118). A pesar de que hasta el momento el trabajo en el propio hogar no ha tenido ninguna consideración jurídica a efecto de generar prestaciones sociales, se ha apuntado "la posibilidad de reconocerle cierta cobertura a efectos de Seguridad Social, o de propiciar su transformación directa en trabajo productivo mediante su externalización» (Grau y Rodríguez, 2015: 107), recuperando así el objetivo - en su día abandonado- de la Disposición adicional primera de la Ley 24/1997, de 15 de julio, de Consolidación y Racionalización del Sistema de Seguridad Social, que pretendía incorporar a la Seguridad Social a las mujeres que «trabajen al cuidado de su propio hogar y no estén amparados por otras prestaciones contributivas». Dotar de caracteres de productividad al trabajo realizado en el hogar familiar, profesionalizándolo, permitiría «aflorar millones de puestos de trabajo e integrar dicho trabajo, que hasta ahora no contabiliza para medir el PIB de cada país, en los regímenes de cotización a los sistemas de protección Social» (Ramos, 2017a: 251 y 552).

\section{MEDIDAS PARA COMBATIR LA BRECHA DE GÉNERO EN LOS USOS DEL TIEMPO: LA RACIONALIZACIÓN DE HORARIOS Y LA GESTIÓN FLEXIBLE DEL TIEMPO DE TRABAJO}

Las pautas tradicionales de organización del tiempo de trabajo en el mercado de trabajo español agravan el conflicto trabajo-familia y las desigualdades por razón de género. Según los datos del MESS correspondientes a 2017, aproximadamente la mitad de los padres empleados tienen turnos partidos con pausas largas para comer, y solo el 15\% de ellos declaran tener control sobre sus horarios de trabajo. Esta situación se combina con una fuerte cultura del presentismo existente en nuestro país que amplía las horas de trabajo de muchos progenitores, especialmente los varones. De ello resulta un panorama con un sistema de horarios disfuncional, que exige de modo urgente la racionalización real de horarios laborales (Mercader y De la Puebla, 2016: 802 y ss.) en consonancia con la demanda de la Asociación para la Racionalización de los Horarios Españoles (ARHOE). Tal propuesta se halla a la espera del futuro Pacto Nacional para la conciliación y la racionalización de horarios que promueva cambios en legislación laboral con el fin de que se flexibilicen y racionalicen los horarios laborales para hacerlos compatibles con el cuidado de los menores y familiares dependientes.

Junto a esta necesidad, se hace igualmente inaplazable avanzar hacia un nuevo modelo de entorno de trabajo. Se trata de que las empresas se conviertan en entornos de trabajo family-friendly que permitan el ejercicio de derechos o ajustes favorables a la conciliación, coadyuvando así a establecer los patrones deseados de parentalidad compartida y, con ello, a alcanzar mayores niveles de igualdad en el mercado de trabajo (Rodríguez González, 2017: 119 y ss.). De forma concreta, este modelo requiere la implantación efectiva del derecho a la gestión flexible del 
tiempo de trabajo, concretada en acuerdos o pactos individuales que garanticen la «vertiente presencial del derecho a la conciliación» (Ballester, 2012: 4), reforzándose así la permanencia laboral femenina como un instrumento para la mayor eficiencia empresarial.

La adopción de medidas de flexibilidad en la jornada de trabajo dentro de las empresas se ha venido reivindicando como uno de los instrumentos de mayor utilidad para hacer efectiva la posibilidad de que los trabajadores compatibilicen su actividad laboral con las responsabilidades de la vida cotidiana, planteándose de forma indirecta en la Estrategia Europa 2020, al señalar como uno de sus objetivos la promoción de «nuevas formas de equilibrio entre la vida laboral y familiar», expresión ésta que parece reforzar la obligación empresarial de reorganización del trabajo para facilitar la compatibilidad entre el trabajo y el adecuado atendimiento de las labores de cuidado. En esta línea se promueve desde la UE la formulación de los derechos de ajuste de jornada vinculados a la reincorporación tras las licencias de maternidad y paternidad. Así, en la cláusula 6 de la Directiva 2010/18/UE ${ }^{15}$ se establecen ciertas prerrogativas del trabajador/a para adaptar su jornada de trabajo con motivo de la reincorporación tras el permiso parental, dando cabida a la aplicación de fórmulas de trabajo flexible relacionadas con el tiempo de trabajo, como la flexibilidad de la jornada laboral, facilitando con ello el mantenimiento de los vínculos familiares tras su reintegración (Cuesta, 2011: 358; Ballester, 2010: 57). Esta previsión presenta, con todo, dos matices negativos que merecen ser valorados como carencias de la política de conciliación de la UE: en primer lugar, el hecho de que este derecho a la adaptación de jornada aparezca limitado exclusivamente a las personas que se reincorporan a la empresa tras un permiso parental, sin que exista un reconocimiento general del derecho a la adaptación de jornada por razones de conciliación ${ }^{16} ; y$, en segundo lugar, es una medida destinada exclusivamente al cuidado de menores, siendo necesaria su ampliación facilitándose el cuidado de cualquier familiar dependiente.

Las mencionadas carencias parecen haberse convertido en una prioridad para la UE. De hecho, la Comisión Europea ha destacado la necesidad de generalizar y fortalecer el derecho a solicitar acuerdos de tiempo de trabajo flexible para personas con responsabilidades familiares, generalizando en el entorno de la UE las fórmulas ya existentes en varios países europeos para ayudar a los trabajadores con el fin de que tengan un mejor equilibrio entre la vida laboral y familiar, permitiéndoles que puedan ocuparse de forma más armónica de sus responsabilidades laborales y familiares, sin dejar de apreciar los posibles beneficios que la flexibilidad puede reportar a las compañías y a la productividad. En este sentido, el art. 9 de la Propuesta de Directiva relativa a la conciliación de la vida familiar y la vida profesional

${ }_{15}$ Directiva 2010/18/UE del Consejo, de 8 de marzo de 2010, por la que se aplica el Acuerdo marco revisado sobre el permiso parental, celebrado por BUSINESSEUROPE, UEAPME, el CEEP, y la CES.

${ }_{16}$ STJUE de 16 de junio de 2016, asunto Rodríguez Sánchez. 
de los progenitores y los cuidadores $^{17}$ amplía el ámbito de actuación de las fórmulas de trabajo flexible abarcando ahora a «los trabajadores con hijos de hasta una edad determinada, que será como mínimo de doce años, y los cuidadores», a quienes se ofrecen las posibilidades de horario de trabajo reducido (reducción de jornada, trabajo a tiempo parcial), calendario laboral flexible y trabajo a distancia.

La traslación de estos objetivos europeos al ordenamiento jurídico español no se ha logrado plasmar de una manera acabada. En su momento, la Ley Orgánica 3/2007, de 22 de marzo para la igualdad efectiva de mujeres y hombres (LOIEMH), atendiendo a la demanda social de dotar de mayores márgenes de flexibilidad en la gestión del tiempo de trabajo por los trabajadores con responsabilidades familiares y asumiendo la idea de que las políticas de work-life balance o de individual flexitime demuestran su beneficio tanto para los trabajadores como para los empresarios, favoreciendo asimismo una mayor presencia femenina en el trabajo productivo (Chacartegui, 2006: 1117), introduce el apartado número 8 en el art. 34 LET -conforme a lo establecido en el Disposición adicional decimoprimera, tres de la LOIEMH-, que reconoce el derecho del trabajador a adaptar la jornada de trabajo para hacer efectivo el derecho a la conciliación de la vida familiar y laboral, en los términos que se establezcan en la negociación colectiva o de conformidad con el acuerdo individual entre el trabajador y el empresario, debiendo respetar lo previsto en el convenio. Desde su incorporación, la redacción del primer párrafo del art. 34.8 LET había sido criticada por su escasa ambición legislativa al no contemplar una serie de derechos específicos, como el de adaptación flexible de la jornada o el de elección de turno de trabajo en razón de la conciliación del trabajo con responsabilidades familiares, aunque se remitieran igualmente a la negociación colectiva los términos de ejercicio de los mismos. En un intento de avanzar en esta línea, con mayor alcance y centrándose en el objetivo de la flexiseguridad, se realiza la reforma operada por la Ley 3/2012, de 6 de julio, de medidas urgentes para la reforma laboral, que añade un segundo párrafo al referido precepto instando a la promoción de ambiciosos instrumentos para la compatibilidad que requieren reordenaciones globales de la estrategia empresarial de recursos humanos, pasando por alto que no todas las empresas admiten mecanismos de compatibilidad como los referidos. De hecho, la incorporación de estos mecanismos en determinadas organizaciones parece improbable, máxime cuando dicha implantación exija a la empresa asumir en solitario el coste derivado de su implantación. Por lo demás, el art. 34.8 LET omite formas menores de ajuste -adaptación de jornada, reducción de jornada en cómputo superior al diario, cambio de turno, etc.- que no implican necesariamente un coste para el empresario y que resultan muy efectivas para facilitar la compatibilidad (Rodríguez, 2016: 175). En este punto conviene resaltar el papel fundamental que está desempeñando la jurisprudencia al combatir las anquilosadas resistencias empresariales a favorecer la conciliación en casos tales como la elección de turno fijo en supuestos de reducción de jornada para el cuidado de

${ }_{17}$ COM (2017) 253 final, 2017/0085(COD) y COM (2017) 252 final. 
hijos o familiares dependientes ${ }^{18}$, tachándolas de actuaciones empresariales discriminatorias (Poyatos, 2018). De este modo, jueces y magistrados se convierten, al juzgar el derecho a conciliar familia y trabajo con perspectiva de género, en piezas fundamentales de la «educación igualitaria» de la que se ve necesitado el tejido empresarial en nuestro país.

Con todo, el derecho a la adaptación de la jornada se configura como un derecho inacabado y cuya determinación precisa y desarrollo pormenorizado debe ser emprendido de forma inmediata por el legislador espańol, sin perjuicio de que, hasta que ello ocurra, sea imprescindible el recurso a la negociación colectiva (donde se pueden configurar fórmulas como las bolsas de horas o los bancos de tiempo de conciliación), o a los acuerdos individuales trabajador-empresario que permitan implantar y generalizar buenas prácticas favorecedoras de la configuración de un entorno laboral sensible a estas necesidades (Mercader y De la Puebla, 2016: 802-806). En todo caso, tales acuerdos deben incluir la existencia de medidas de acción positiva que rompan la tradicional resistencia masculina -fundamentada en el estereotipo ideal del empleado perpetuamente disponible- a hacer ajustes relacionados con el empleo ante la paternidad.

\section{UNA PARENTALIDAD POSITIVA PARA UNA IGUALDAD EFECTIVA}

Otro mecanismo para compensar la brecha de género en el mercado laboral lo constituyen las medidas orientadas a la consecución de la corresponsabilidad en la asunción de las labores de cuidado. Y ello sobre la base de que «la implicación paterna en el cuidado de los hijos es un factor clave [...] para lograr una mayor igualdad de oportunidades entre mujeres y hombres» (Flaquer y Escobedo, 2014: 70). Así lo refleja el Consejo de la Unión Europea en las Conclusiones tituladas Respuesta al compromiso estratégico para la igualdad de género de la Comisión ${ }^{19}$, donde indica que "los hombres han de desempeñar un papel fundamental para garantizar que se avance hacia la igualdad de género, en particular a través de un reparto equitativo de las responsabilidades del cuidado de las personas dependientes, uno de los requisitos previos para la participación igualitaria de las mujeres en el mercado laboral».

De este modo, se considera necesario apostar por un diseño de los permisos parentales cuyas características aumenten el equilibrio en el uso por parte de ambos sexos (Castro y Pazos, 2012: 9). La consecución de este objetivo exige configurar los derechos de conciliación como derechos de titularidad individualizada (Cabeza, 2010: 52; Lewis et al., 2008: 274), así como promover una serie de actuaciones com-

\footnotetext{
${ }_{18}$ Entre otras, STSJ de Canarias/Las Palmas, de 15 de diciembre de 2017 (rec. 1249/2017).

19 Adoptadas por el Consejo EPSCO en su sesión n. ${ }^{\circ}$ 3474, celebrada el 16 de junio de $2016(10416 / 16)$.
} 
plementarias, a saber: a) la supresión de los derechos de conciliación femeninos; b) la creación de derechos masculinos de paternidad o de cuotas de reserva masculinas en derechos de conciliación de titularidad neutra; c) el reconocimiento de derechos individuales a cada progenitor sin posibilidad de transferencia al otro progenitor; y d) las acciones positivas dirigidas a la asunción masculina de cargas familiares (Lousada, 2011: 5).

Para que el ejercicio de la parentalidad positiva ${ }^{20}$ se garantice de manera efectiva resulta imprescindible que los marcos institucionales y legislativos permitan que tanto las mujeres como los hombres dispongan de una verdadera libertad de elección personal en la conciliación de la vida profesional, familiar y privada como forma de hacer avanzar la consecución práctica de la igualdad de facto entre hombres y mujeres. Este aspecto tiene, sin duda, una especial repercusión en la vertiente de la titularidad de los permisos parentales, pues los marcos actuales, heredados de un diseño basado en la óptica de la garantía de la igualdad formal, redundan en la persistencia de diferencias prácticas en las cifras de utilización masculina y femenina de los permisos parentales (OIT, 2016).

En este sentido, con el objetivo de lograr una mayor participación de los padres en el cuidado de los menores, la Resolución europea del Consejo y de los Ministros de Trabajo y Asuntos Sociales de 29 de junio de 2000, relativa a la participación equilibrada de hombres y mujeres en la actividad profesional y en la vida familiar, alentaba a los Estados miembros a evaluar la posibilidad de que los respectivos ordenamientos jurídicos reconocieran a los hombres trabajadores un derecho individual e intransferible al permiso de paternidad, manteniendo sus restantes derechos laborales y sociales, correspondiendo a los propios Estados determinar las formas en orden a la concesión de ese derecho.

Los progresos experimentados en los últimos años en la formulación de la titularidad de los derechos parentales han pretendido continuar avanzando hacia la corresponsabilidad en la asunción de las obligaciones familiares ${ }^{21} \mathrm{y}$, en consecuencia, se ha apostado por la individualización de los derechos de conciliación (Lewis, 2006: 274). Así, tomando como premisa que la presencia del padre en la vida del menor constituye un valor importante en el desarrollo físico, emocional y psicológico saludable de éste ${ }^{22}$, la Directiva 2010/18 establecía la intransferibilidad de al menos

${ }^{20}$ Recomendación Rec (2006)19 del Comité de Ministros a los Estados Miembros sobre políticas de apoyo al ejercicio positivo de la parentalidad (adoptada por el Comité de Ministros el 13

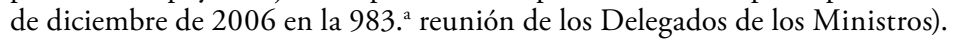

${ }^{21}$ Considerando 12 de la Directiva 2010/18/UE.

22 Así lo expresa el Segundo Informe de la Comisión de Derechos de la Mujer e Igualdad de Género sobre la propuesta de Directiva del Parlamento Europeo y del Consejo por la que se modifica la Directiva 92/85/CEE del Consejo, relativa a la aplicación de medidas para promover la mejora de la seguridad y de la salud en el trabajo de la trabajadora embarazada, que haya dado a luz o en periodo de lactancia (A7-0032/2007), de 5 de marzo de 2010, p. 57. También es una idea apoyada por la Resolución Europea del Consejo y de los Ministros, de 29 de junio de 2000, sobre participación equilibrada de hombres y mujeres en la actividad profesional y en la vida familiar (art. 16 de la Directiva 2006/54/CE). 
un mes, del permiso parental mínimo de cuatro meses que configura, con el fin de que sirva de «incentivo positivo para que lo utilicen los padres» (considerando 16) y que se pretende reforzar con el contenido del art. 4 de la Propuesta de Directiva relativa a la conciliación de la vida familiar y la vida profesional de los progenitores y los cuidadores $^{23}$, que, a modo de norma mínima para el permiso de paternidad a nivel de la UE, introduce el derecho de los padres a acogerse al permiso de paternidad durante un breve periodo, que no debe ser inferior a diez días laborables, con ocasión del nacimiento de un hijo. Esta previsión ha sido objeto de pronunciamientos e interpretaciones divergentes. Para algunos autores, esta cláusula pretende dar cabida a nuevos modelos de familias, como las monoparentales (Cabeza, 2010). Otras voces, por su parte, apuestan por un planteamiento que incida directamente en el reparto de los tiempos, valorando la posibilidad de que la norma hubiese limitado la transferibilidad de un mes de duración «sólo en relación con la madre, en caso de existir progenitores de distinto sexo» (Rodríguez Rodríguez, 2010: 87).

Con la finalidad de acomodar la legislación española a las orientaciones europeas y que la conciliación de la vida personal, familiar y laboral se desarrolle en términos de corresponsabilidad, desde la entrada en vigor de la LOIEMH, estableció una nueva causa de suspensión del contrato de trabajo en el Estatuto de los Trabajadores $-1995^{24}$-, en razón de la paternidad, que se complementó con la inclusión en el marco de la acción protectora de la Seguridad Social con una prestación económica homónima que establece una renta que viene a sustituir el salario dejado de percibir durante la suspensión de la actividad laboral al cesar temporalmente la prestación de servicios (arts. 183-185 del Real Decreto Legislativo 8/2015, de 30 de octubre, por el que se aprueba el Texto refundido de la Ley General de la Seguridad Social).

De este modo, en el texto del vigente Estatuto de los Trabajadores (arts. 45.1.d) y 48.7 del Real Decreto Legislativo 2/2015, de 23 de octubre), el permiso por paternidad español se configura como derecho de titularidad individual y de disfrute intransferible a la suspensión del contrato de trabajo durante cuatro semanas (Lousada, 2008: 28; Albiol, 2007: 100 y ss.). La regulación de este derecho, que «se reconoce tanto en los supuestos de paternidad biológica como en los de adopción y acogimiento" (Beltrán de Heredia, 2008: 324), ha sido alabada por la utilización de términos genéricos que permiten el acceso al derecho a realidades familiares alternativas al modelo tradicional de familia.

Ahora bien, el resto de permisos parentales (excedencias, reducciones de jornada, etc.) siguen estando formulados como derechos de titularidad neutra o indiferenciada, lo cual redunda en que sigan siendo las mujeres las que continúen ejerciendo o disfrutando de tales derechos, asumiendo las consecuencias que ello implica de abandono del trabajo (siquiera temporal) en el desarrollo de las carreras

${ }^{23}$ COM (2017) 253 final, 2017/0085(COD) y COM (2017) 252 final.

${ }^{24}$ Real Decreto Legislativo 1/1995, de 24 de marzo, por el que se aprueba el texto refundido de la Ley del Estatuto de los Trabajadores. 
profesionales, en su promoción profesional, en la renta disponible para la unidad familiar y, en su caso, en el volumen de las prestaciones futuras de seguridad social y en su independencia económica durante la vejez. Un ejemplo particularmente llamativo al respecto lo constituyen las reducciones de jornada dado que tan solo un $4 \%$ de los padres de al menos un niño/a menor de ocho ańos reduce su jornada para cuidar frente a una de cada cuatro madres.

Este doble abordaje en la titularidad de los derechos de conciliación -titularidad individual para el permiso de maternidad y maternidad, y titularidad indistinta para el resto de permisos parentales- ha venido a ser calificado como «modelo mixto" por el Parlamento Europeo ${ }^{25}$ considerándose, al menos en teoría, un modelo adecuado, pues permite que los progenitores puedan decidir conjuntamente y de la forma más adecuada cómo gestionar sus permisos en el mejor interés de los hijos y teniendo en cuenta las particularidades de sus respectivos empleos. Sin embargo, los datos ponen de manifiesto que, a pesar de que los permisos parentales se configuran de manera indistinta y están a disposición de ambos progenitores, la tasa de participación en los permisos parentales es de un $10 \%$ en el caso de los varones frente a un 97\% de las mujeres (OECD, 2016).

Estos datos muestran cómo difícilmente se consigue el objetivo de la corresponsabilidad a través del reconocimiento de derechos de titularidad neutra, es decir, con derechos teóricamente ejercitables por hombres y mujeres, puesto que la neutralidad de su formulación no elimina la presunción de que este tipo de derechos sigan siendo disfrutados mayoritariamente por las mujeres. Para romper esta tendencia, el Pilar Europeo de Derechos Sociales ${ }^{26}$, proclamado de forma solemne el 17 de noviembre de 2017 en la Cumbre Social en favor del empleo justo y el crecimiento de Gotemburgo, pone especial énfasis en el hecho de que no se ofrecen a los hombres posibilidades ni alicientes suficientes para hacer uso de los permisos y ello afianza el papel de las mujeres como cuidadoras primarias, con los consiguientes efectos negativos sobre el empleo femenino. En definitiva, se considera indispensable la adopción de medidas eficaces y adecuadas de incentivación de la utilización masculina de este tipo de medidas, por ejemplo, el reconocimiento de ayudas o subvenciones económicas a favor de los hombres que hagan uso de los mismos. En este ámbito han desempeñado un papel fundamental el conjunto de medidas de incentivación adoptadas en el ámbito autonómico (Martínez, 2011: 245-269; y Rodríguez González, 2010: 59 y ss.).

25 Resolución del Parlamento Europeo, de 12 de mayo de 2016, sobre la aplicación de la Directiva 2010/18/UE del Consejo, de 8 de marzo de 2010, por la que se aplica el Acuerdo marco revisado sobre el permiso parental (2015/2097(INI)).

${ }^{26}$ Comunicación de la Comisión al Parlamento Europeo, al Consejo, al Comité Económico y Social Europeo y al Comité de las Regiones «Establecimiento de un pilar europeo de derechos sociales». COM (2017) 250 final, y cuyo antecedente más inmediato es la Resolución del Parlamento Europeo de 27 de enero de 2017 (2016/2095(INI). Texto disponible en https://ec.europa.eu/commission/sites/beta-political/files/social-summit-european-pillar-social-rights-booklet_en.pdf. 
Con todo, la medida verdaderamente revolucionaria procorresponsabilidad que actuaría en la médula de la desigualdad de género y de los problemas de conciliación sería la equiparación del permiso de paternidad con el de maternidad (Gala, 2017: 93). Éste es el sentido de la Proposición no de ley aprobada el pasado 18 de octubre de 2016 por el Pleno del Congreso de los Diputados, que insta al Gobierno a estudiar esta reforma que pretende igualar en 16 semanas los permisos de maternidad y paternidad configurando un «Sistema de Permisos Parentales 16+16», donde cada uno de los progenitores disponga de 16 semanas de permiso no transferibles, remunerados y con amplio margen de flexibilidad en el disfrute.

\section{REFLEXIONES FINALES}

La incorporación y permanencia de las mujeres en el mercado de trabajo se enfrenta al enquistado problema de las brechas de género en el ámbito de las relaciones laborales que se manifiestan en distintas esferas o ámbitos: acceso al empleo; salarios y pensiones; ascenso de las mujeres a puestos de liderazgo y especial responsabilidad; y usos del tiempo y conciliación de la vida laboral familiar.

La lucha contra las desigualdades y discriminaciones en el mercado laboral se ha implantado como una idea fuerza en las políticas internacionales, europeas y nacionales cristalizándose en un conjunto de mecanismos y medidas destinadas a combatir las distintas manifestaciones de la brecha de género en el mundo del trabajo y alcanzar, con ello, niveles adecuados de igualdad efectiva. No obstante, parece razonable concluir que el abanico de medidas articuladas hasta el momento es insuficiente para paliar los efectos de las mencionadas brechas de género, persistiendo en el mundo del trabajo diferencias injustificadas de valoración y tratamiento de la aportación que realizan las mujeres a la sociedad (Ramos, 2017a: 245).

En este sentido, junto a la importancia de la generalización de las medidas de acción positiva y articulación de derechos laborales efectivos y «específicos de las mujeres» (Casas, 2017: 2) como mecanismos reparadores, se concibe imprescindible reforzar el papel activo de las políticas de conciliación como sistema de catapulta hacia un mercado laboral más igualitario (Rodríguez González, 2017b), especialmente: a) perfeccionando mecanismos de conciliación en la vertiente de la ordenación y organización del tiempo de trabajo que posibiliten el equilibrio trabajo-familia sin tener que renunciar a la "presencia» en el mercado de trabajo (horario flexible con adaptación de la hornada de trabajo); b) favoreciendo medidas de gestión flexible del tiempo de trabajo (trabajo a distancia, teletrabajo); c) promoviendo la utilización masculina de los permisos parentales atribuyéndoles en exclusiva determinados derechos laborales de conciliación; d) equiparando la duración de los permisos parentales de titularidad individualizada e intransferible (maternidad y paternidad); e) dotando de cobertura y valor económico al trabajo no remunerado de los cuidados; f) promoviendo medidas de concienciación y sensibilización que coadyuven a la necesaria transformación social en el reparto de las responsabilidades de cuidado y de las tareas domésticas superando los estereotipos tradicionales de género y el modelo tradicional de división sexual del trabajo; y, finalmente, g) reforzando la 
corresponsabilidad social a través del incremento de programas públicos y la mejora den la accesibilidad y la calidad de la red de servicios públicos de atención a menores y familiares enfermos o dependientes.

Recibido: 28-2-2018; aceptado: 21-6-18 


\section{BIBLIOGRAFÍA}

Albiol Montesisnos, I. (2007). «Suspensiones y excedencias», en I. Albiol Montesinos; C.L. Alfonso Mellado y J.F. Blasco Lahoz, Los aspectos laborales de la Ley de Igualdad. Valencia: Tirant lo Blanch, pp. 100-120.

Ballester Pastor, M.A. (2010). "Conciliación laboral y familiar en la Unión Europea: rémoras, avances y nuevas líneas de tendencia en el ámbito de la corresponsabilidad». Revista de Derecho Social, núm. 51, pp. 43-46.

Ballester Pastor, M.A. (2012). «Conciliación y corresponsabilidad en las últimas reformas laborales y de seguridad social». Disponible en http://www.iuslabor.org/wp-content/plugins/ download-monitor/download.php?id=173.

Beltrán De Heredia Ruiz, I. (2008). «La suspensión de la relación de trabajo por maternidad y por paternidad a la luz de la LO 3/2007». Relaciones Laborales, núm. 1, pp. 305-324.

Cabeza Pereiro, J. (2010). «¿En qué debe cambiar el derecho español para adaptarse al acuerdo marco revisado sobre el permiso parental?». Aranzadi Social, núm. 6, Estudio (BIB 2010/1242), edición online no paginada.

Casas Baamonde, M.E. (2017). «¿Derechos fundamentales específicos de las mujeres?». Revista de las Relaciones Laborales, núm. 1, pp. 1-18.

CC. OO. (2018). La brecha salarial, factor de quiebra democrática. Confederación Sindical de CC. OO.

CES- EsPaña: Informe 01/2011, Tercer informe sobre la situación de las mujeres en la realidad sociolaboral española. Madrid.

Chacartegui Jávega, C. (2006). «Tiempo de trabajo, racionalidad horaria y género: un análisis en el contexto europeo». Relaciones Laborales, núm. 2, pp. 1201-1123.

Comisión Europea (2013). The Gender Gap in Pensions in the European Union. Luxemburgo: Oficina de Publicaciones de la Unión Europea.

Comisión Europea (2014). Cómo combatir la brecha salarial entre hombres y mujeres en la Unión Europea. Luxemburgo: Oficina de Publicaciones de la Unión Europea.

Consejo de la Unión Europea (2015). Conclusiones del Consejo sobre Igualdad de oportunidades de ingresos para las mujeres y hombres: cerrando la brecha de género en las pensiones, 19 de junio de 2015 [SOC 371, GENDER 7, PENS 4].

Cuesta López, V. (2011). «Derechos de conciliación de la vida personal, familiar y profesional: fundamentos constitucionales y avances normativos en el ámbito de la Unión Europea». Revista de Derecho Constitucional Europeo, núm. 6, pp. 331-376.

EIGE (2017). Gender Equality Index 2017: Measuring gender equality in the European Union 2005-2015, Oficina de publications de la UE, disponible en http://eige.europa.eu/ rdc/eige-publications/gender-equality-index-2017-measuring-gender-equality-european-union-2005-2015-report.

EUROFOUND (2016). The gender employment gap: challenges and solutions. Luxemburgo: Oficina de Publicaciones de la UE.

Fernandez-Lozano, I. (2017). "If you dare to ask: self-perceived possibilities of Spanish fathers to reduce work hours». Community, Work \& Family, DOI: 10.1080/13668803.2017.1365692.

FMI (2013). Las mujeres, el trabajo y la economía: beneficios macroeconómicos de la equidad de género. Departamento de Estrategia, Políticas y Evaluación y departamento de Finanzas Públicas. 
Flaquer, L. y Escobedo, A. (2014). «Licencias parentales y política social de la paternidad en España». Cuadernos de Relaciones Laborales, núm. 32 (1), 69-99.

Gala Durán, C. (2017). «El permiso por paternidad: un balance tras casi diez años después de su implantación». Revista de Trabajo y Seguridad Social. Centro de estudios Financieros, núm. 406, pp. 57-94.

Grau Pineda, C. y Rodríguez González, S. (2015). Conciliación y Seguridad Social. La brecha de género en el Sistema de Pensiones. Valencia: Tirant lo Blanch.

Lewis, J., Knijn, T., Martin, C. y Ostner, I. (2008). «Patterns and developments in work/family recognition policies for parents in France, Germany, the Netherlands and the UK in the 2000s». Social Politics: International Studies in Gender, State \& Society, núm. 15, pp. 261-286.

Lousada Arochena, J.F. (2011). «La individualización de los derechos de conciliación. Una asignatura pendiente». Themis, Revista Jurídica de Igualdad de Género, núm. 9, pp. 5-10.

Lousada Arochena, J.F. (2008). Permiso de paternidady conciliación masculina. Albacete: Bomarzo.

Martínez YÁŃEZ, N.M. (2011). «El principio de corresponsabilidad en las políticas autonómicas de conciliación», en J. Cabeza Pereiro y B. Fernández Docampo (dirs.), Conciliación de la vida familiar y laboral y corresponsabilidad entre sexos. Valencia: Tirant lo Blanch, pp. 245- 269.

Mercader Uguina, J. R., y De la Puebla Pinilla, A. (2016). "Capítulo XVI. Políticas de Preservación y de Reparto del Empleo (I)», en J.L. Monereo Pérez, J.A. Fernández Bernat y B. López Insúa (dirs. y coords.), Las Politicas Activas de Empleo: Configuración y Estudio de su Regulación Jurídica e Institucional. Navarra: Thomson Reuters Aranzadi, pp. 793-811.

OCDE (2013). Cerrando las brechas de género: es hora de actuar, CIEDESS.

OCDE (2016). Parental leave: where are the fathers? París: OECD Publishing.

OIT (2013). Ante el centenario de la OIT: realidades, renovación y compromiso tripartito. Memoria del Director General. Confederación Internacional del Trabajo. 102. ${ }^{a}$ reunión. Informe 1 (A). Ginebra: Oficina Internacional del Trabajo.

OIT (2015). La mujer en la gestión empresarial: cobrando impulso. Ginebra: Oficina Internacional del Trabajo.

OIT (2016). Women at work. Trends 2016. Ginebra: Oficina Internacional del Trabajo.

Poyatos i Matas, G. (2018). "Juzgando con perspectiva de género el derecho a conciliar familia y trabajo. A propósito de la Sentencia de la Sala Social del Tribunal Superior de Justicia de Canarias/Las Palmas de 15 de diciembre de 2017 (rec. 1249/2017)». RTSS. CEF, núm. 420, pp. 1-12.

Ramos Martín, N.E. y Blázquez Cuesta, M. (2007). "Contrato de trabajo a tiempo parcial en los Países bajos: ¿sólo para mujeres?». Revista del Ministerio de Trabajo y Asuntos Sociales, núm. 66, pp. 143-159.

Ramos Quintana, M.I. (2015). «Las mujeres y el futuro del trabajo: las iniciativas de la OIT». Trabajo y Derecho, núm. 4, pp. 99-103.

Ramos Quintana, M.I. (2017a). «El futuro de las mujeres. El futuro de la humanidad; más derechos efectivos para un empoderamiento real», en El futuro del trabajo que queremos. Conferencia nacional tripartita. Madrid: MESS, pp. 243-258.

Ramos Quintana, M.I. (2017b). «Concentración de la riqueza y desigualdad salarial». Trabajo y Derecho, núm. 27, pp. 9-13. 
Rodríguez GonZÁlez, S. (2010). «La corresponsabilidad como eje de las políticas públicas de conciliación de la vida familiar y laboral. Marcos comparados autonómicos». Revista de Trabajo y Seguridad Social. Centro de Estudios Financieros, núm. 333, pp. 59-108.

Rodríguez GonzÁlez, S. (2016). Tiempo de trabajo y vida privada. Granada: Comares.

Rodríguez GonzÁlez, S. (2017a). «Trabajo decente y riesgos psicosociales: la organización del tiempo de trabajo», en M.I. Ramos Quintana (coord.), Riesgos psicosociales y organización en la empresa. Navarra: Aranzadi, pp. 97-144.

Rodríguez GonzÁLEz, S. (2017b). «Desigualdad por causa de género en la Seguridad Social: carreras de cotización y prestaciones». Lan harremanak: Revista de relaciones laborales, núm. 38, pp. 93-125.

Rodríguez-Piñero y Bravo-Ferrer, M. (2004). «El informe sobre el impacto por razón de género en la elaboración de las disposiciones normativas». Relaciones Laborales, núm. 10, pp. 117-128.

Rodríguez Rodríguez, E. (2010). Instituciones de conciliación de trabajo con el cuidado de familiares. Albacete: Bomarzo.

Tinios, P., Bettio, F. y Betti, G. (2015). Men, women and pensions. Luxemburgo: Oficina de Publicación de la Unión Europea.

Tortuero Plaza, J.L. (2006). «Conciliación, género y empleo: reflexiones desde el Sistema de la Seguridad Social», Revista del Ministerio de Trabajo y Asuntos Sociales, núm. 1, pp. 113-130. 
